\title{
Comment l'école reste inégalitaire. Comprendre pour mieux réformer
}

Hugues Draelants, Presses universitaires de Louvain, Louvain-la-Neuve, $2018,186 \mathrm{p}$.

Jean-Marie De Ketele

\section{CpenEdition}

\section{Journals}

Édition électronique

URL : https://journals.openedition.org/ries/8129

DOI : $10.4000 /$ ries. 8129

ISSN : 2261-4265

Éditeur

France Education international

Édition imprimée

Date de publication : 1 avril 2019

Pagination : 40-44

ISBN : 978-2-85420-623-4

ISSN : $1254-4590$

Référence électronique

Jean-Marie De Ketele, «Comment l'école reste inégalitaire. Comprendre pour mieux réformer », Revue internationale d'éducation de Sèvres [En ligne], 80 | avril 2019, mis en ligne le 01 avril 2019, consulté le 24 juin 2021. URL : http://journals.openedition.org/ries/8129 ; DOI : https://doi.org/10.4000/ries.8129

Ce document a été généré automatiquement le 24 juin 2021.

(c) Tous droits réservés 


\section{Comment l'école reste inégalitaire. Comprendre pour mieux réformer}

Hugues Draelants, Presses universitaires de Louvain, Louvain-la-Neuve, 2018, 186 p.

Jean-Marie De Ketele

\section{RÉFÉRENCE}

Comment l'école reste inégalitaire. Comprendre pour mieux réformer, Hugues Draelants, Presses universitaires de Louvain, Louvain-la-Neuve, 2018, 186 p.

1 Chercheur au GIRSEF (Groupe interfacultaire de recherche sur la socialisation, l'éducation et la formation) de l'Université catholique de Louvain, H. Draelants tente de comprendre, dans une première partie de l'ouvrage, pourquoi l'école reste inégalitaire, dans un contexte où la démocratisation l'a rendue accessible à tous et où les pouvoirs politiques, notamment sous la pression des évaluations internationales, affirment vouloir réduire les inégalités. À partir d'un bilan de quinze années de recherche, l'auteur va développer deux hypothèses explicatives qui structurent l'ouvrage « si les inégalités perdurent c'est d'abord qu'elles se transforment continuellement »; «la reproduction des inégalités tient aussi à ce qu'on peine à réformer l'école ».

2 La question des inégalités scolaires est classique en sociologie. Le lecteur est donc en droit de se demander s'il va trouver une énième synthèse des travaux connus sur le sujet. Si c'est bien le cas pour le premier chapitre, c'est que l'auteur en avait besoin pour se distancier des positions sociologiques classiques et démontrer que les mécanismes producteurs des inégalités scolaires avaient évolué dans un triple sens: (a) une recomposition horizontale des inégalités, qui implique de reconsidérer la distinction entre inégalités de réussite et inégalités d'orientation ; (b) un renforcement du rôle de l'établissement avec une intrication plus étroite qu'auparavant entre les facteurs familiaux et scolaires; (c) une transformation du rôle du capital culturel dans 
la reproduction, dont non seulement le contenu évolue, mais aussi le mode de transmission, qui devient plus actif et indirect.

3 Les deux chapitres suivants illustrent une transformation fondamentale, selon H. Draelants: on passe de l'image bourdieusienne de l'héritier à celle de l'initié. L'héritier était l'étudiant privilégié dont les succès scolaires étaient dus à la transmission, par héritage familial, d'un capital culturel scolairement valorisé. Or, dit l'auteur, la rentabilité de la culture générale classique est en déclin au profit de nouvelles hiérarchies disciplinaires. À une transmission culturelle "osmotique » et passive fait place une transmission culturelle active et indirecte de la part des familles; celles-ci assurent un suivi scolaire étroit, recourent au besoin à des cours particuliers, et développent, dès le début de la scolarité, des stratégies pour amener leurs enfants dans les filières élitistes. Ces élèves ne sont plus nécessairement des héritiers, mais des initiés. Ainsi, ils sont nombreux chez les enfants d'enseignants, dont les parents maitrisent l'information sur les conséquences du choix d'un établissement scolaire et ont les moyens économiques nécessaires pour envoyer leurs enfants dans les établissements élitistes des centres villes. Les initiés se trouvent aussi dans les familles diplômées de l'enseignement supérieur, chez les indépendants aisés ou les cadres d'entreprise, car ils allient la maîtrise de l'information et le capital économique. Hugues Draelants reprend à Brown (1990) le concept de "parentocratie » pour désigner ces initiés.

4 Dans le chapitre 4, l'auteur commence par définir le concept de mérite comme un ensemble de qualités intellectuelles (intelligence) et morales (ardeur au travail, sens de l'effort) socialement valorisées et attribuées à l'individu. Tout au long de leur scolarité, ces élèves initiés seront généralement caractérisés comme faisant preuve de mérite ; et ils auront donc accès ultérieurement aux positions de pouvoir, après avoir été scolarisés dans les établissements élitistes qui ouvrent les portes. Faut-il ou non promouvoir la méritocratie, c'est-à-dire une société dans laquelle l'accès aux positions de pouvoir est censé avant tout dépendre du mérite ? La question mérite réflexion, car suite aux transformations des mécanismes de production des inégalités scolaires, la méritocratie semble correspondre actuellement à la parentocratie. Aussi faut-il, selon l'auteur, conduire une politique qui initie les «non-héritiers », si l'on veut réduire les inégalités scolaires.

5 Pourquoi l'égalité des chances méritocratiques n'est-elle pas obtenue, malgré les intentions affichées par les réformes successives? Telle est la question de la seconde partie de l'ouvrage.

6 L'auteur tient d'abord à distinguer changement et réforme. La réforme, dit-il, est un projet de changement de type radical, enclenché et donc voulu par les autorités éducatives en vue de modifier le système scolaire et, en principe, d'en améliorer le fonctionnement. Les réformes échouent souvent, mais cela ne veut pas dire que l'école ne change pas : la première partie de l'ouvrage a montré que les inégalités ne changent pas, mais que de nouvelles modalités de reproduction se développent. Dubet et Merle (2016) expliquent cet échec par deux composantes. La première tient au fait que les acteurs sociaux ont toujours une marge de manœuvre à l'intérieur de contraintes collectives; la seconde estime que les autorités politiques conçoivent leurs réformes sur la base d'un diagnostic incomplet et de modèles théoriques implicites. H. Draelants va tenter d'approfondir et de compléter cette double explication en prenant appui, cette fois, sur des travaux qu'il a menés en Belgique sur deux tentatives de réformes, 
l'une visant à supprimer le redoublement (chapitre 6), l'autre cherchant à imposer un système d'inscription des élèves au début du secondaire, afin de réguler la composition sociale des établissements (chapitres 7 et 8). Notons que ces réformes ont été largement soutenues par les chercheurs en éducation, sur la base d'arguments à la fois scientifiques et idéologiques.

7 L'auteur commence par battre sa coulpe de chercheur et celle de ses collègues, en dénonçant leurs habitudes de pensée: leurs travers intellectualistes les amènent à penser qu'il suffit de mettre en place des politiques d'éducation en cohérence avec les résultats de leurs recherches; et leurs travers conflictualistes ne leur permettent pas de comprendre les résistances des acteurs sociaux (enseignants, chefs d'établissement, familles), qu'ils assimilent à la seule poursuite par ces derniers de leurs intérêts. Cette prise de conscience l'amène à revisiter la littérature scientifique sur le redoublement et à constater que les effets du redoublement sont très différents selon les modalités réelles de fonctionnement du système éducatif.

8 Les entretiens avec les enseignants et les chefs d'établissement mettent en évidence qu'ils ne sont pas opposés au principe du non-redoublement, mais que le cadre de fonctionnement qui est le leur les conduit à des difficultés insurmontables pour eux : gérer l'hétérogénéité de plus en plus grande des élèves au fil du temps; motiver tous les élèves et pas seulement quelques-uns; mettre en place des dispositifs efficaces de différenciation dans le contexte de la classe; ne pas abaisser le niveau d'attentes sous peine de se voir mis sous pression par les familles et les politiques de reddition de comptes. L'auteur en conclut qu'il ne suffit pas d'un changement culturel des acteurs chargés de mettre en place la réforme, mais que ce changement culturel n'est possible que s'il s'accompagne d'un changement structurel, ce qui amène à s'interroger sur bien des aspects du fonctionnement, tels que la logique méritocratique, l'évaluation certificative, la concurrence entre établissements et la logique du quasi-marché, et bien d'autres.

9 Il faut donc passer de la politique des bonnes intentions, prégnante dans le monde éducatif, à une politique qui prenne en compte l'expertise non profane des acteurs de terrain et comprendre les fonctions latentes qui structurent les pratiques et le fonctionnement réel d'un établissement scolaire, en sachant que tous les établissements n'ont pas nécessairement le même fonctionnement s'ils ne sont pas confrontés aux mêmes difficultés.

Cette position va être confortée par l'analyse des réformes successives menées en Belgique pour imposer un système bureaucratique d'inscription, dont l'intention était de réduire les inégalités scolaires en réduisant l'homogénéité sociale des établissements scolaires. Auparavant, le choix d'une école secondaire s'opérait grâce à une relation personnalisée entre l'école et la famille, l'une exposant le projet qui caractérise l'identité de l'établissement, l'autre faisant part des caractéristiques et des besoins de l'enfant ainsi que des attentes des parents. Cette relation disparaît avec le nouveau système bureaucratique (une procédure algorithmique de traitements des données mentionnées dans le formulaire rempli par les parents). Mais les initiés s'emploient à déjouer les effets de la réforme en s'informant non seulement sur les critères utilisés dans le traitement, mais aussi sur les caractéristiques des différents établissements et leurs hiérarchisations en termes de réputation dans leur réseau social, en s'arrangeant pour que leurs enfants et ceux du même réseau social se retrouvent dans un même établissement et permettent un entre-soi, et enfin en en palliant les éventuels 
inconvénients liés à l'hétérogénéité par le recours à l'école de l'ombre. Malgré les tentatives du législateur pour déjouer les stratégies des initiés, les inégalités scolaires persistent.

11 L'échec d'une réforme est-elle finalement une fatalité ? Pas nécessairement, dit Draelants. Les autorités éducatives ont souvent de bonnes raisons de proposer une réforme et les acteurs sociaux d'aussi bonnes raisons de la refuser dans leurs pratiques quotidiennes. Il faut donc s'y intéresser pour comprendre le fonctionnement réel du système éducatif et pouvoir ainsi offrir des changements structurels progressifs, qui permettront des changements culturels. Il s'agit donc plutôt de concevoir un processus incrémental et progressif de changements qui procèdent par petites touches et résulte d'interactions entre acteurs et organisations scolaires. S'agit-il encore de réforme, au sens où l'auteur avait défini ce concept? Peut-être, si le projet radical de changement est conçu comme un processus à long terme qui sera pensé en termes d'articulations et de séquençages des politiques éducatives, pour rendre possible leur mise en œuvre. Le temps politique étant un temps court, peut-on encore parler de réformes possibles? Comme nous le montrait le colloque organisé par cette revue (Revue internationale d'éducation de Sèvres, $\mathrm{n}^{\circ}$ 68), seuls quelques systèmes éducatifs ont réussi à raisonner dans un temps long grâce à une grande stabilité politique et de l'administration éducative.

Ce sont là des questions fondamentales que la lecture de ce bel ouvrage, très bien construit et rédigé clairement, nous amène à nous poser, quelques semaines après le colloque organisé par la revue en juin 2019 sur le thème des « conditions de réussite des réformes en éducation $» .^{1}$ Une lecture à encourager.

\section{NOTES}

1. https://journals.openedition.org/ries/6658

\section{AUTEUR}

\section{JEAN-MARIE DE KETELE}

Jean-Marie De Ketele est professeur émérite de l'Université catholique de Louvain (Belgique) et de l'Université Cheikh Anta Diop de Dakar, où il a créé la Chaire Unesco en sciences de l'éducation (1994). Docteur honoris causa de plusieurs universités, il a présidé l'Association internationale de pédagogie universitaire ainsi que l'Association pour le développement des méthodologies de l'évaluation en éducation (ADMEE-Europe). Ses travaux portent principalement sur la pédagogie universitaire, sur l'évaluation des apprentissages et des 
systèmes éducatifs ainsi que sur l'engagement professionnel des acteurs de l'éducation et de la formation. Il dirige plusieurs collections d'ouvrages scientifiques aux Éditions De Boeck et est membre du conseil scientifique de la Revue internationale d'éducation de Sèvres. Courriel : jeanmarie.deketele@uclouvain.be 